\title{
Implicit LES of turbulent, separated flow: wall-mounted hump configuration
}

\author{
Susheel Sekhar* and Nagi N. Mansour ${ }^{\dagger}$ \\ NASA Ames Research Center, Moffett Field, CA 94035 \\ David Higuera Caubilla ${ }^{\ddagger}$ \\ Institut Supérieur de l'Aéronautique et de l'Espace, Toulouse, France
}

\begin{abstract}
Direct simulations (ILES) of turbulent, separated flow over the wall-mounted hump configuration is conducted to investigate the physics of separated flows. A chord-based Reynolds number of $\operatorname{Re}_{c}=47,500$ is set up, with a turbulent inflow of $\operatorname{Re}_{\theta}=1,400(\theta / c$ $=3 \%)$. FDL3DI, a code that solves the compressible Navier-Stokes equations using highorder compact-difference scheme and filter, with the standard recycling/rescaling method of turbulence generation, is used. Two different configurations of the upper-wall are analyzed, and results are compared with both a higher $\mathbf{R e}_{c}\left(=\mathbf{9 3 6 , 0 0 0 ,} \mathbf{R e}_{\theta}=\mathbf{7 , 2 0 0 , \theta / c = 0 . 7 7 \% )}\right.$ experiment for major flow features, and RANS (k- $\omega$ SST) results. A lower $\mathbf{R e}_{c}$ allows for DNS-like mesh resolution, and an adequately wide span. Both ILES and RANS show delayed reattachment compared to experiment, and significantly higher skin friction in the forebody of the hump, as expected. The upper-wall shape influences the $C_{p}$ distribution only. Results from this study are being used to setup higher $\mathbf{R e}_{c}$ (lower $\theta / c$ ) ILES.
\end{abstract}

\section{Introduction}

Accurate prediction of turbulent flows at high Reynolds numbers continues to be a significant problem in fluid dynamics. When turbulent boundary layers separate, predictions become all the more challenging since our understanding of the underlying physics is still lacking. Currently, most turbulent flows in engineering applications are simulated using various models that represent the understood physics. There is still no universal model that can be used to compute every turbulent flow scenario.

Various workshops over the past decade have brought together CFD practitioners to assess current capabilities of different types of turbulent flow solution methodologies, and use benchmarked experiments to identify potential areas of improvement. In 2004, the Langley Research Center Workshop on CFD Validation of Synthetic Jets and Turbulent Separation Control ${ }^{1,2,3}$ (CFDVAL2004) considered three flows of interest that had been studied experimentally, and had extensive statistical databases of mean and turbulent quantities. Case 3 at the workshop was flow over the wall-mounted hump configuration, which consisted of a Glauert-Goldschmied-type airfoil mounted between two glass end-plates. It is a nominally two-dimensional experiment, although there are three-dimensional side-wall effects near the end-plates. This is based on earlier experiments of Seifert and Pack, ${ }^{4}$ who had studied flows over a similar configuration at higher Reynolds numbers $\left(\operatorname{Re}_{c}=2.4-2.6 \times 10^{6}\right.$, based on chord-length). The baseline experiment that was used as the reference in the workshop did not involve flow control, and was conducted by Greenblatt et al. ${ }^{5}$ It had an $\operatorname{Re}_{c}$ of 936,000, with a turbulent inflow $\operatorname{Re}_{\theta}=7,200$ at $\mathrm{M}_{\infty}=0.1$. Both leading and trailing edges were faired smoothly with a wind tunnel splitter plate, whereas the experiments of Seifert and Pack installed the airfoil on the wind tunnel floor. This baseline experiment without flow-control showed that flow separates near $65 \%$ of the chord, and reattaches downstream past the hump. Other than $C_{p}$ and $C_{f}$ distributions, $2 \mathrm{D} /$ stereo PIV data of velocity profiles and turbulence quantities along the tunnel center plane were documented.

At the workshop, this case was analyzed extensively. Numerous RANS (Reynolds-averaged NavierStokes) studies were conducted and all failed to reproduce the many characteristics of this flow. Iaccarino et

* NASA Postdoctoral Program Fellow, AIAA Member

${ }^{\dagger}$ Chief Division Scientist, NASA Advanced Supercomputing Division, AIAA Associate Fellow

${ }^{\ddagger}$ Double Masters Student, SUPAERO-Universidad Politécnica de Madrid 
al. ${ }^{6}$ used CIRA ${ }^{\mathrm{a}}$-UZEN ${ }^{\mathrm{b}}$ and the commercial code Fluent to test the Spallart-Allmaras and k- $\omega$ turbulence models, and could not predict this baseline flow with satisfactory agreement. Capizzano et al. ${ }^{7}$ used a modified version of CIRA-UZEN, and tested the Menter-SST k- $\omega$ model. They found that the Reynolds stresses downstream of the point of separation were under-predicted, and reattachment therefore delayed. Cui and Agarwal ${ }^{8}$ used an unsteady RANS solver (WIND) and the SST model, and found the combination to severely over-predict the surface pressure. This also resulted in a longer separation bubble. Each of these results used a second-order structured-grid methodology to compute the flow. Balakumar ${ }^{9}$ used a fifth-order WENO (weighted essentially non-oscillatory), with third-order TVD (total variation diminishing) RungeKutta, and the SST model. Morgan et al. ${ }^{10}$ used second- and fourth-order compact-difference scheme and the $\mathrm{k}-\varepsilon$ turbulence model for the case. Both resulted in a similar under-prediction of the Reynolds stresses, and a longer bubble. Bettinni and Cravero, ${ }^{11}$ He et al. ${ }^{12}$ and Madugundi et al. ${ }^{13}$ used Fluent with different turbulence models. Bettinni and Cravero used the RNG k- $\varepsilon$ model, and found it to significantly under-predict surface pressure over the attached portion of the hump, and a very rapid rise in pressure in the separated region. He et al. used the k- $\varepsilon, \mathrm{S}-\mathrm{A}$, and SST models, and made significantly better pressure predictions using the same software. S-A and SST models still over-predicted the separation bubble size, and the k- $\varepsilon$ model predicted later separation. Madugundi et al. surprisingly under-predicted the size of the separation bubble using the same software, with a number of turbulence models. The all-pervading observation with RANS models is the inconsistency of the size of the separation bubble, attributed to eddy-viscosity and Reynolds stresses being under-predicted within the separation region. Rumsey ${ }^{14}$ used CFL3D, a URANS code, with three different turbulence models, and showed that doubling eddy-viscosity in a region near the separation bubble did indeed result in earlier reattachment, more comparable to experiment.

Some LES and hybrid RANS/LES methods made much better predictions of the flow features. Biswas ${ }^{15}$ used an incompressible fifth-order WENO code with upwind-biased finite differencing to solve the LES equations. In this, sub-grid scale turbulent stresses were closed using a dynamic turbulent kinetic energy transport model. A spanwise width of $0.2 c$ ( $c$ - chord length) was set with periodic boundaries. Very good agreement of surface pressure and skin friction were seen with experiment. Krishnan et al. ${ }^{16}$ conducted RANS simulations (using S-A and SST), as well as DES of the hump flow problem, and showed that DES could improve the bubble size prediction, reducing it by $20 \%$ compared to RANS. The velocity profiles compared well with experiment, but the Reynolds shear stress $\langle u v\rangle$ was slightly over-predicted. Their DES was setup with a periodic span of $0.121 c$. Šarić et al. ${ }^{17}$ solved the incompressible RANS, LES and DES equations using a cell-centered collocated finite-volume scheme, with the SIMPLE algorithm. They used the S-A model for RANS and for the unresolved scales in DES $\left(\Delta z^{+}=150\right)$, and the Smagorinsky sub-grid scale model for LES with a grid spacing of $\Delta x^{+}=80, \Delta y^{+}=1, \Delta z^{+}=50$ at the wall. The spanwise widths were $0.2 c$ for DES and $0.152 c$ for LES (which was found to be insufficient to ensure spanwise decorrelation), with periodic boundary conditions. In spite of the coarseness, LES showed very good comparisons with experiment in terms of shear stresses and bubble length. DES performed similarly well, and the S-A RANS simulation once again over-predicted the bubble length. You et al. ${ }^{18}$ performed incompressible LES with an energy-conservative second-order central difference scheme on a staggered mesh, where aliasing errors were controlled by enforcing kinetic energy conservation. This was done with the dynamic Smagorinsky sub-grid scale model. A recycling inflow procedure was used on a mesh with a spanwise extent of $0.2 c$, and a wall resolution of $\Delta x^{+} \leq 50, \Delta y^{+} \leq 0.8, \Delta z^{+} \leq 25$. Surface pressure, mean velocity profiles and Reynolds stresses compared very well with experiment. Skin friction in the fore-body of the airfoil was over-predicted. But, the size of the separation bubble agreed well with experiment. Franck and Colonius ${ }^{19}$ conducted compressible LES and ILES with a non-density weighted low-pass filtered method, with a sixth-order Padé scheme in the wall-normal direction, fourth-order explicit treatment in the streamwise direction, and a Fourier spectral method in the spanwise direction. They used a filtering optimization parameter of $\alpha_{f}=0.45$. The mesh resolution was $\Delta x^{+} \leq 94, \Delta y^{+} \leq 8.7, \Delta z^{+} \leq 31$, with a spanwise extent of $0.2 c$. A much finer resolution was imposed over the hump, and within the separation region. Overall, the velocities, Reynolds stresses and the bubble length agreed well with experiment. They compared results using both constant coefficients and dynamic Smagorinsky, as well as using no sub-grid scale model, and found very little difference. They also analyzed results at higher Mach numbers: $\mathrm{M}_{\infty}=0.1$ and 0.3 were identical, whereas $\mathrm{M}_{\infty}=0.6$ showed higher acceleration over the hump, and a longer separation bubble.

There were other hybrid RANS/LES that did not improve on the RANS results during the workshop.

${ }^{a}$ Centro Italiano Ricerche Aerospaziali

${ }^{\mathrm{b}}$ Unsteady Zonal Euler Navier-Stokes 
Israel et al. ${ }^{20}$ used the blended RANS/LES method flow simulation methodology (FSM) in a secondorder code based on flux difference splitting and dual time-stepping. The underlying RANS model used was an explicit algebraic stress model (EASM) based on $\mathrm{k}-\varepsilon$. With a span of $0.17 c$, the time-averaged results showed a reattachment point too far downstream, similar to RANS. Hiller and Seitz ${ }^{21}$ used the scale adaptive simulation (SAS) blended RANS/LES model on a domain with $0.56 c$ span. Their limited quantitative comparisons showed pressure distribution similar to the k- $\omega$ RANS model, with much higher pressure within the separation region than experiment.

Morgan et al. ${ }^{22}$ conducted compressible ILES with a fourth-order compact-difference scheme, and used a high-order non-dispersive Padé-type filter instead of a sub-grid scale model. Their method was similar to that of Franck and Colonius, with $\alpha_{f}=0.4$ in a sixth-order filter. To attain a reasonable resolution $\left(\Delta x^{+}=60, \Delta y^{+}=1, \Delta z^{+}=20\right)$, they scaled down $\operatorname{Re}_{c}$ to 200,000 on a mesh with a spanwise width of $1.39 c$. This corresponded to a momentum thickness to chord ratio $(\theta / c)$ of $3.6 \%$. The mean velocity profiles compared well except near the point of reattachment, and the suction pressure was higher than experiment, as was skin friction in the fore-body of the hump. These were attributed to the lower Reynolds number used in the simulation. Upstream of the separation point, Reynolds stresses were significantly over-predicted, and it should be noted that for this lower Reynolds number, the separation point was further upstream compared to experiment.

Postl and Fasel $^{23}$ conducted the only known DNS for this case (using the incompressible vorticity-velocity form of the Navier-Stokes equations), with a mesh resolution that resulted in the simulation being underresolved $\left(\Delta x^{+}=27-92, \Delta y^{+}=1.2, \Delta z^{+}=17\right)$, and a span of $0.142 c$. In addition to being under-resolved, a thinner laminar inflow profile with $\operatorname{Re}_{\theta}=4,000$ at $x / c=-0.5$ was used. This is equivalent to a $\operatorname{Re}_{\theta}=$ 2,400 at $x / c=-2.14(\theta / c=0.26 \%)$. This was tripped near the inflow using a time-harmonic forcing term. Even with that setup, the separation point and mean velocity profile before separation matched well with experiment. But the size of the separation bubble and reattachment point were both over-predicted by about $20 \%$. This was attributed to the relative coarseness of the mesh, which failed to resolve the smallest scales adequately. Further, lack of information about freestream turbulence intensity added to these discrepancies, particularly in the range of frequencies where the separated shear layer is hydrodynamically unstable.

Besides CFDVAL2004, more recent workshops have also included this test case to further assess the state-of-the-art in CFD methods. Both the 11th and the 12th ERCOFTAC EAHR $^{\mathrm{d}}$ Workshops on Refined Turbulence Modelling had results of flow over the hump-flow configuration. ${ }^{24,25}$ This test case has since been added to the ERCOFTAC Database (Classic Collection) as Case C.83 ${ }^{\mathrm{e}}$. It was also included in the ECCOMAS CFD 2010 Conference as a part of the V\&V I: Code Verification and Solution Verification \& Validation Workshop ${ }^{\mathrm{f}}$, and the Advanced Turbulence Simulation for Aerodynamic Application Challenges (ATAAC) project ${ }^{g}$. Further interest in this test case has even led to lattice Boltzmann simulations of the configuration, coupled with a very large eddy simulation (VLES) turbulence model. Noelting et al. ${ }^{26}$ used a 19-state lattice Boltzmann model (D3Q19), with the collision integral in the Boltzmann equation being approximated using the Bhatnagar-Gross-Krook (BGK) form. Turbulence modeling was directly included in the lattice Boltzmann equations by modifying the relaxation time, to give an extended relaxation time scale. This extended time was then calculated using a variant of the RNG $\mathrm{k}-\varepsilon$ model. This resulted in the emergence of significantly more complex physics from the RNG k- $\varepsilon$ equations, than when using the Navier-Stokes equations. Results were similar to DES and LES in terms of surface pressure and bubble length.

Other LES and hybrid RANS/LES methods have also been studied in more detail since the workshop, and many efforts have shown very good progress and excellent comparisons with experiment, ${ }^{27,28,29,30}$ especially with DDES. ${ }^{31,32,33}$ But, the significant shortcomings of RANS models are yet to be addressed. Yoshio and Abe $^{34}$ and Cappelli and Mansour ${ }^{35}$ recently documented these failures, including the under-prediction of the Reynolds stresses within the separation region, a farther reattachment location, and a slower recovery of the boundary layer after reattachment.

Seifert and Pack $^{4}$ noted that for the given fully turbulent inflow conditions, the characteristics of separation and the bubble length were largely insensitive to the inflow $\operatorname{Re}_{\theta}$ and $\operatorname{Re}_{c}$. This was especially true since laminar to turbulent transition was eliminated in the domain. But the ratio of the thickness of the incoming

\footnotetext{
${ }^{\mathrm{c}}$ European Research Community on Flow, Turbulence And Combustion

$\mathrm{d}$ The International Association for Hydro-Environment Engineering and Research

ehttp://cfd.mace.manchester.ac.uk/ercoftac/

f www.maretec.ist.utl.pt/html_files/VV2010.htm

ghttp://cfd.mace.manchester.ac.uk/twiki/bin/view/ATAAC/TestCase002WallHumpDescription
} 
boundary layer to the hump height $(\delta / h)$ played a significant part. In other words, matching experimental $\theta / c$ is critical in replicating the conditions for similar flow separation. Post and Fasel, ${ }^{23}$ on the other hand, attributed differences between their DNS and experiment to the coarseness of their mesh, and a narrow span. Given the computational costs of setting up DNS at the experimental conditions (estimated to be upwards of 6 billion grid points with the current setup), a preliminary test case of lower $\operatorname{Re}_{\theta}: \operatorname{Re}_{c}$ flow over the wall-mounted hump is considered. In compromising on the $\theta / c$ ratio, a mesh resolution comparable to DNS is realizable, as is a wide-enough span. This is conducted as a preliminary step towards setting up subsequent simulations at experimental $\theta / c$, in addition to lending an extra data-point, considering the lack of high quality DNS for this configuration. RANS simulations using the k- $\omega$ SST model at this $\operatorname{Re}_{\theta}: \operatorname{Re}_{c}$ combination are also conducted. Comparisons of pressure and skin friction, as well as velocity and Reynolds stress profiles are made with the available experimental data with a lower $\theta / c$.

This paper is organized as follows: Section II briefly explains the numerical methodologies used for the ILES. Section III presents the results, which include the turbulent inflow generation using the recycling/rescaling method, as well as flow over the wall-mounted hump geometry. Qualitative and quantitative comparisons are made with experiment, as well as with RANS. Conclusions are drawn in Sec. IV.

\section{Numerical Method}

\section{II.A. Governing Equations, Compact Difference Scheme and Filter}

The finite-difference code (a variant of AFRL's FDL3DI ${ }^{36,37}$ ) used in this study solves the compressible Navier-Stokes equations for an ideal gas. The sixth-order compact-difference scheme of Lele ${ }^{38}$ is used to solve the governing equations in transformed curvilinear coordinates. ${ }^{39,40}$ Fourth-order explicit Runge-Kutta (RK-4) is used for time integration. During each time step, an eighth-order low-pass spatial filtering scheme is applied to the conservative variables to ensure stability, along with second- and fourth-order near-boundary formulations. ${ }^{41}$ A filtering optimization parameter of $\alpha_{f}=0.495$ is set. This results in lesser dissipation compared to the setups used by Morgan et al. ${ }^{22}\left(\alpha_{f}=0.4\right)$ and Franck and Colonius ${ }^{19}\left(\alpha_{f}=0.45\right)$. To ensure stability and accuracy of time integration, a Courant-Friedrichs-Lewy (CFL) number in the vicinity of 0.4 , based on the acoustic speeds, is used in the RK-4 scheme.

This code was previously designed to solve for supersonic flows, specifically those involving shock-wave and boundary layer interaction problems. Therefore, the localized artificial diffusivity (LAD) method of shock-capturing was previously installed by Kawai et al., ${ }^{42,43}$ and was used in a detailed study of the flow physics of such interactions by Morgan et al. ${ }^{44}$ This method adds artificial coefficients to the fluid transport terms, and takes effect in the vicinity of shocks. Since the flow regime under consideration is subsonic, LAD is not included in the current study. Thus, other than the low-pass filter, no additional sub-grid scale model is used. In that sense, this effort can be considered implicit-LES, or more precisely "under-resolved DNS."

\section{II.B. Recycling/Rescaling Turbulence Inflow Method}

For generating the turbulent inflow, the standard recycling/rescaling method of Lund et al. ${ }^{45}$ is used in the code. Urbin and Knight ${ }^{46}$ extended this method to compressible flows for solving a Mach 3 turbulent boundary layer. This was implemented as a part of the effort to study the interaction of an oblique shock wave and a turbulent boundary layer by Morgan et al. ${ }^{47}$ The approach extracts the instantaneous velocity profile from a plane downstream of the inflow, rescales and reintroduces it as the inflow. The schematic of the procedure is shown in Fig. 1. It has been shown to produce a realistic turbulent boundary layer, yielding statistics that are in good agreement with experiment and DNS. To further prevent contamination of the solution with spurious spatio-temporal correlations generated by this reintroduction procedure, a nonconstant reflection of the recycled turbulence plane is applied at randomly distributed time intervals. ${ }^{47}$ This method, called recycling/rescaling with dynamic reflection $(R R+D R)$, has been used to solve subsonic flows here. 


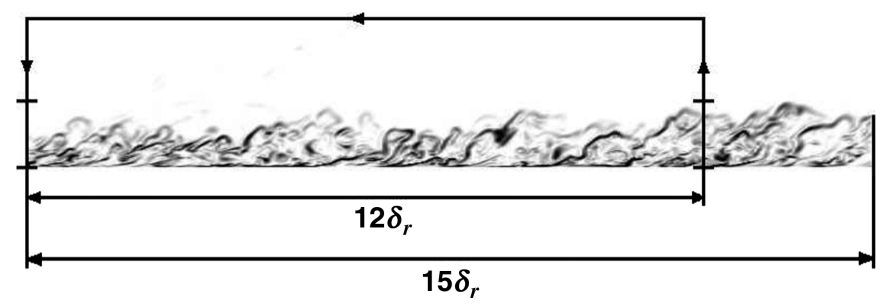

Figure 1. Recycling-rescaling methodology schematic ${ }^{48}$

\section{Results}

\section{III.A. Computational Setup}

Details of the wall-mounted hump simulation are explained here. This is followed by validation of the inflow profile, and the comparison of results of the ILES with RANS and experiment.

As mentioned before, the presented ILES corresponds to a higher $\theta / c$ on a mesh with DNS-like resolution. For the inflow to the hump domain, a turbulent velocity profile at $\mathrm{M}_{\infty}=0.3$ and $\operatorname{Re}_{\theta}=1,400$ was chosen. This is in contrast to the experimental inflow of $\mathrm{M}_{\infty}=0.1$ and $\mathrm{Re}_{\theta}=7,200$. Franck and Colonius ${ }^{19}$ showed that the flow over the hump is insensitive to differences in freestream Mach number, from 0.1 to 0.3 . $\operatorname{Re}_{\theta}$ was so chosen to allow for the relatively inexpensive validation of the inflow profile with the spectral DNS data of Spalart. ${ }^{49}$ With these conditions, a domain of length $15 \delta_{\mathrm{R}}$, height $3 \delta_{\mathrm{R}}$ and span $1 \delta_{\mathrm{R}}$ was used, where $\delta_{\mathrm{R}}$ is the desired boundary layer thickness at the velocity capture-plane. The height of the domain was chosen based on the validation study of Lund et al. ${ }^{45}$ for the same $\operatorname{Re}_{\theta}$. The spanwise width was identified in a parametric study that investigated the minimum width required for turbulent boundary layers to match reference DNS. 50

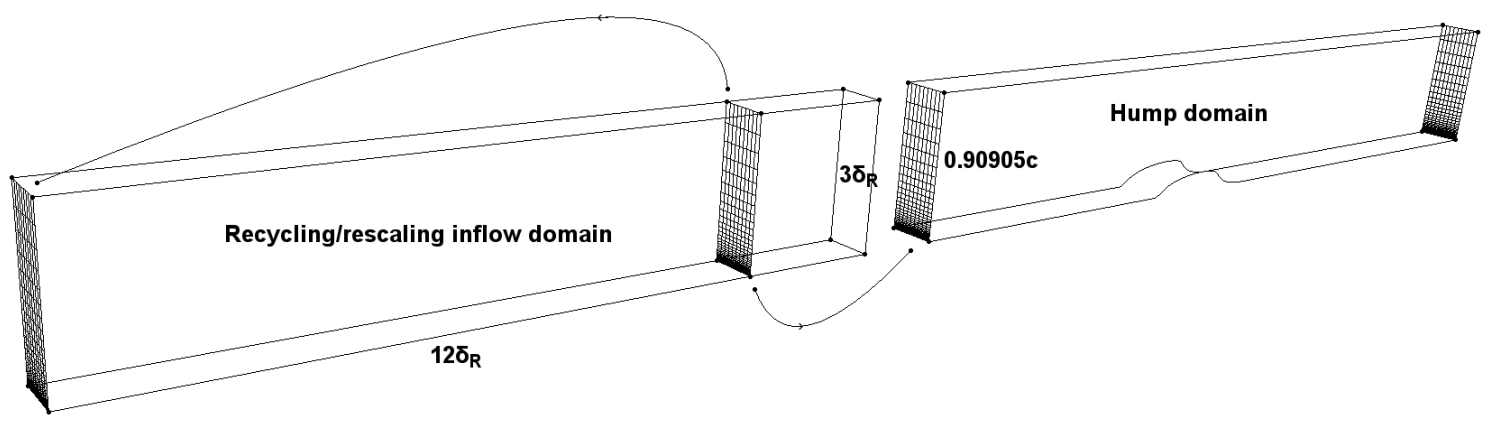

Figure 2. Perspective view of the schematic of the computational setup

The dimensions of the hump domain were defined based on the CFDVAL2004 workshop. The inflow plane was located 2.14 chord-lengths $(c)$ upstream of the leading edge of the hump, and the exit plane $4.0 c$ downstream, respectively. The height of the domain from the splitter-plate to the upper wall was $0.90905 c$. The chord-based Reynolds number was computed based on the constraints set by the dimensions of the inflow and hump domains. Thus, $3 \delta_{\mathrm{R}}=0.90905 c$ (Fig. 2). The theoretical $\delta_{\mathrm{R}}$ for this inflow, based on Prandtl's one-seventh power law, was computed to be $0.002061 \mathrm{~m}$, resulting in a chord of $0.0068 \mathrm{~m}$. The experimental chord length was $0.42 \mathrm{~m}$. With a freestream Mach number of 0.3 , the ILES was setup for an $\operatorname{Re}_{c} \approx 47,500$, which is one-twentieth of the experimental $\operatorname{Re}_{c}(=936,000)$. Thus, the $\theta / c$ ratio was computed to be $3 \%$, compared to an experimental $\theta / c$ of $0.77 \%$. It should be mentioned here that the coarse DNS of Postl and Fasel $^{23}$ had a $\theta / c$ of $0.26 \%$, whereas the lower $\operatorname{Re}_{c}$ ILES of Morgan et al. ${ }^{22}$ had their $\theta / c=3.6 \%$. With such significant differences in flow conditions, major flow features and trends in pressure and skin friction were the main points of comparison in this study, as were the mean velocity profiles and Reynolds stresses. 


\section{III.B. Turbulent Inflow}

The turbulent inflow profile with $\operatorname{Re}_{\theta}=1,400$ was generated simultaneously as an auxiliary simulation using the recycling/rescaling method. ${ }^{45,46,47}$ The inflow capture-plane was located about $12 \delta_{\mathrm{R}}$ downstream of the inlet, and was also the location of the target $\operatorname{Re}_{\theta}$ (Fig. 1). This mesh consisted of $500 \times 100 \times 105$ points, and was uniformly spaced along the streamwise and spanwise directions. A hyperbolic tangent stretching was set in the wall normal direction. With these, the mesh resolution at the wall was comparable to DNS, with $\Delta x^{+}=19.4, \Delta y^{+}=0.65, \Delta z^{+}=6$. This was validated by comparing the mean velocity profile here with the spectral DNS data of Spalart ${ }^{49}$ (Fig. 3).

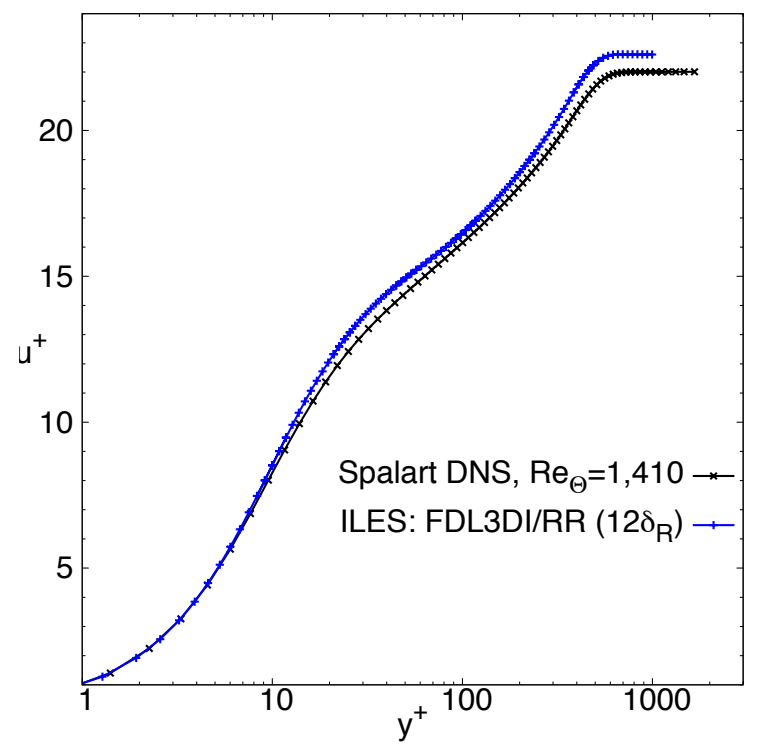

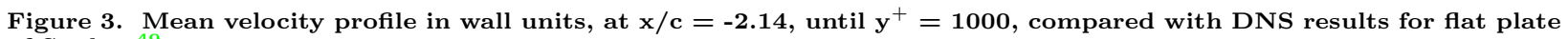
of Spalart ${ }^{49}$

There is very good agreement through the viscous sublayer, and the log layer. The slightly higher freestream velocity at the edge of the boundary layer is attributed to the upper-wall boundary condition. Whereas the spectral DNS corresponds to a zero pressure-gradient flat plate boundary layer, the upper-wall in the inflow domain is set to a symmetry boundary. This results in a slight acceleration of the freestream. The Reynolds stresses at the same $12 \delta$ location are compared with reference spectral DNS in Fig. 4.

Figure 4 (a) compares the Reynolds stresses throughout the boundary layer. Differences are evident within the buffer zone, whilst the rest of the inflow profile compares well with spectral DNS. The overprediction, also evident in the mean velocity profile, is discussed in a separate paper by the authors, ${ }^{50}$ and is once again attributed to differences in the upper-wall boundary condition.

\section{III.C. Wall-mounted Hump}

With the validated inflow profile, ILES of the hump flow was carried out on two separate meshes, shown in Fig. 5. These meshes differed in the shape of the upper-wall. During the CFDLVAL2004 workshop, the top-wall shape was so modified to account for side-wall blockage effects, which improved the agreement of the pressure distribution over the hump with experiment. At this higher $\theta / c$, both upper-wall profiles were tested to document differences at the current flow conditions. "struct1" corresponds to the flat upper-wall configuration, and struct 4 has the modified shape. Each mesh consisted of $827 \times 100 \times 105$ points, with a similar DNS-like resolution at the wall $\left(\Delta x^{+} \leq 19.4, \Delta y^{+} \leq 0.65, \Delta z^{+} \leq 6\right)$. It should be noted here that the coarse DNS of the higher $\mathrm{Re}_{c}$ flow by Postl and Fasel ${ }^{23}$ used a mesh with $\Delta x^{+}=27-92, \Delta y^{+}=1.2, \Delta z^{+}=17$, on a domain of span $0.142 c$. The current setup had a span of $0.3 c$. This setup, therefore, address both drawbacks of the earlier coarse DNS, despite being for a higher $\theta / c$.

For the remaining boundary conditions, a freestream pressure outflow was set at the outlet. No-slip, adiabatic walls were used for the bottom and top walls. Whereas most of the simulations during the workshop 


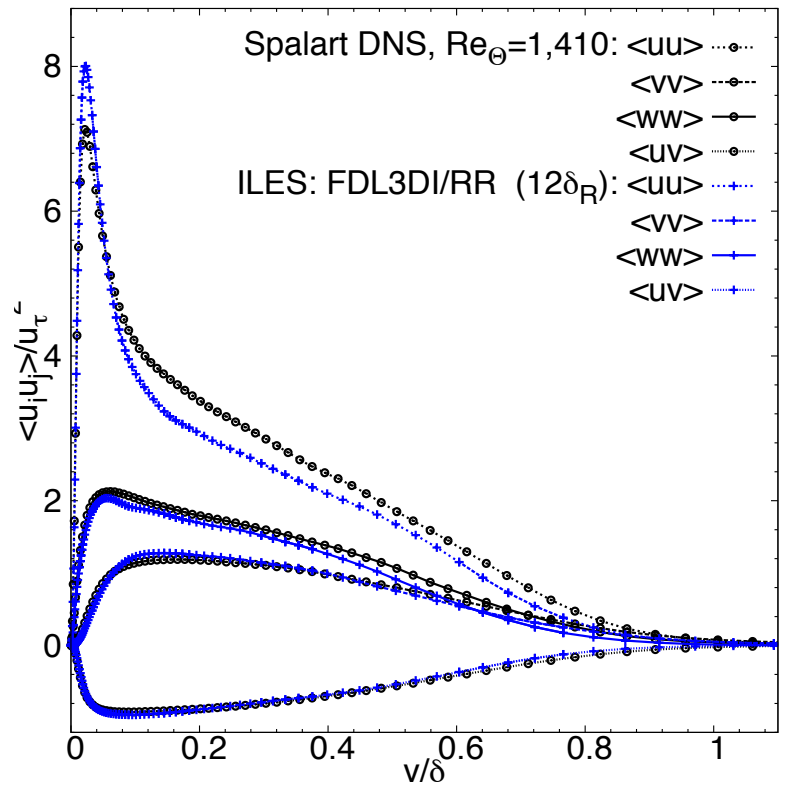

(a) Across the boundary layer

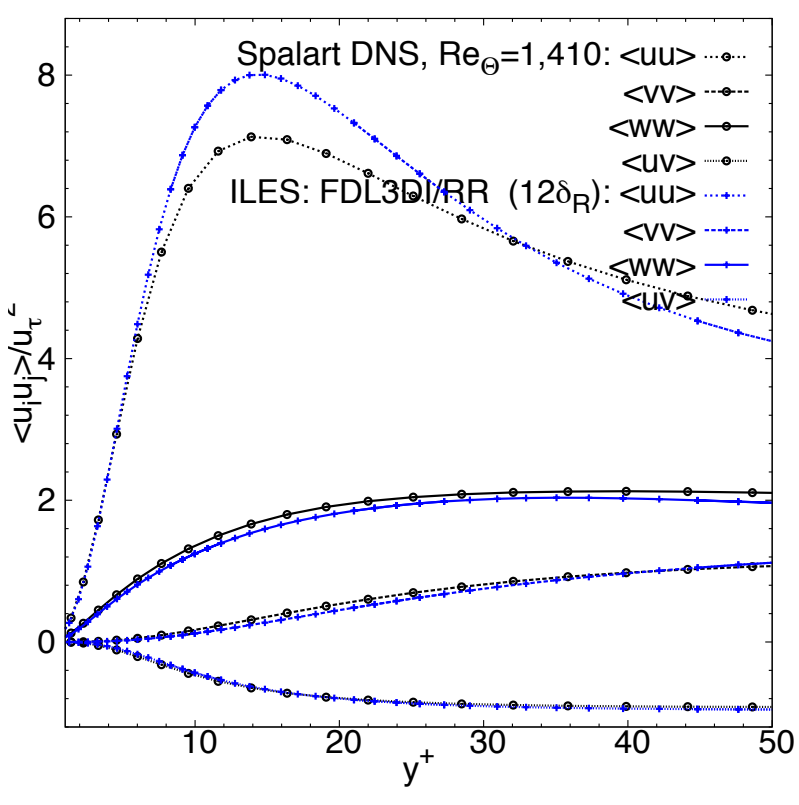

(b) In the viscous near-wall region

Figure 4. Reynolds stresses normalized by friction velocity, at $\mathrm{x} / \mathrm{c}=\mathbf{- 2 . 1 4}$, compared with DNS results of Spalart ${ }^{49}$

used a symmetry or slip-wall boundary at the upper-wall, this was so chosen to be a better representation of the experimental setup. Along the span, periodic boundary conditions were set.

The non-dimensionlized mean velocity contours are shown in Fig. 6. Figure 6 (a) shows the experimental 2D PIV results for the $\operatorname{Re}_{c}=936,000$ case, and Figs. 6 (b) and (c) represent the ILES results for $\operatorname{Re}_{c}=$ 47,500 using meshes "struct1" and "struct4," respectively. The first observation that stands out is the longer separation bubble at the higher $\theta / c(3 \%)$. There is little difference between the two meshes, and both predict the reattachment point downstream of that for the experiment with $\theta / c=0.77 \%$. In addition, the point of separation is further upstream. Both these observations conform with those seen in the ILES of Morgan et al. ${ }^{22}$ whose $\theta / c$ was a comparable $3.6 \%$. The recirculation bubble is also evident in Fig. 7 , which represents the streamlines of the flow, shown for the experiment (a), and ILES ((b) and (c)). The earlier separation and delayed reattachment are both clearly apparent here.

For a quantitive comparison of the flow features, pressure and skin friction distributions along the wall were compared with experiment, and RANS results. OpenFOAM ${ }^{\mathrm{h}}$ (Open source Field Operation And Manipulation), used for the RANS simulations, is a free, open source software suite written in $\mathrm{C}++$ that allows for the development of customized numerical solvers and pre/post-processing tools for solving fluid dynamics or other continuum mechanics problems. Solving turbulent flows requires a Navier-Stokes equations solver that includes a turbulence model to close the Reynolds stress terms. For these RANS simulations, simpleFoam, a steady-state solver for incompressible turbulent flows that employs the Semi-Implicit Method for Pressure Linked Equations (SIMPLE) algorithm was used with the $k-\omega$ SST model. Suitably scaled down versions of CFDVAL2004 workshop meshes (scale $=1 / 20$ ) were used, with a steady freestream inflow set at $x / c=-3$ (instead of $x / c=-6.39$ used at the workshop) to account for the reduced $\operatorname{Re}_{\theta}$ at the inlet. Simulations with both upper-wall shapes were tested, with both slip and no-slip upper-wall boundaries.

Comparisons of $C_{p}$ and $C_{f}$ are shown in Fig. 8. In Fig. 8 (a), pressure over the fore-body of the hump are significantly higher for ILES and RANS than the $\operatorname{Re}_{c}=936,000$ experiment, and lower within the separation bubble. The higher pressure over the fore-body was also seen in the ILES of Morgan et al., but $C_{p}$ within the separation bubble is counter to their observation. Whereas their ILES showed higher pressure within the separation bubble compared to experiment, the current ILES showed an even lower $C_{p}$ within the bubble, as did RANS. Also, in agreement to the cases in the CFDVAL2004 workshop, the modified upper-wall did result in a lower $C_{p}$ over the fore-body section of the hump.

\footnotetext{
$\mathrm{h}_{\mathrm{www} . o p e n f o a m . c o m}$
} 


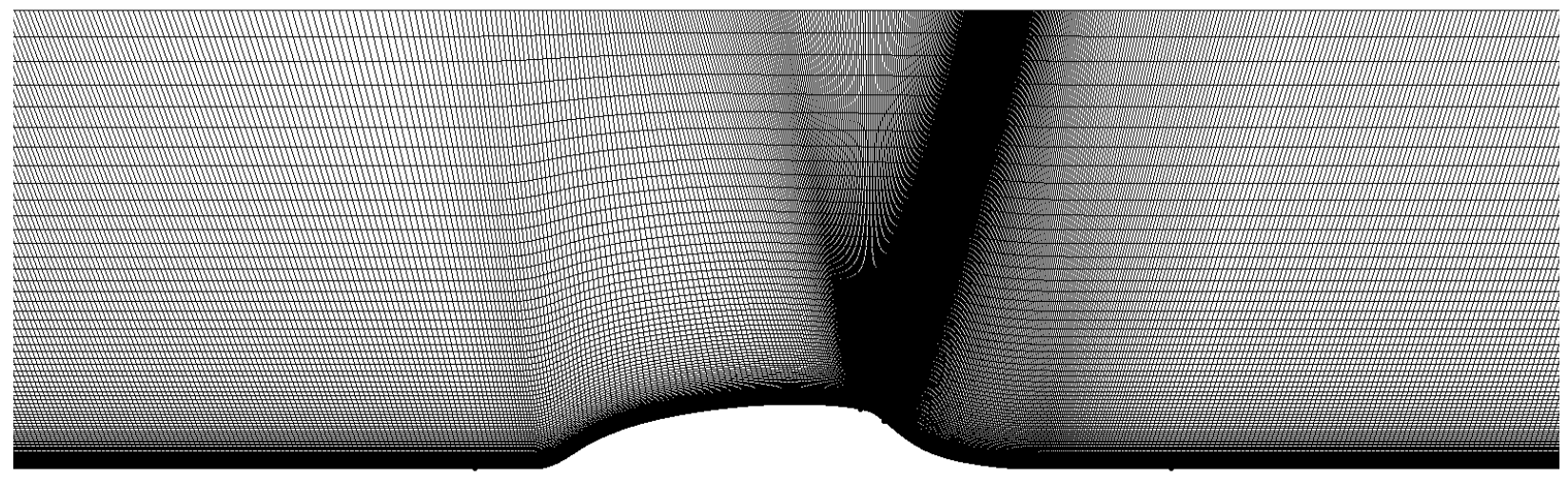

(a) struct1: default flat upper-wall

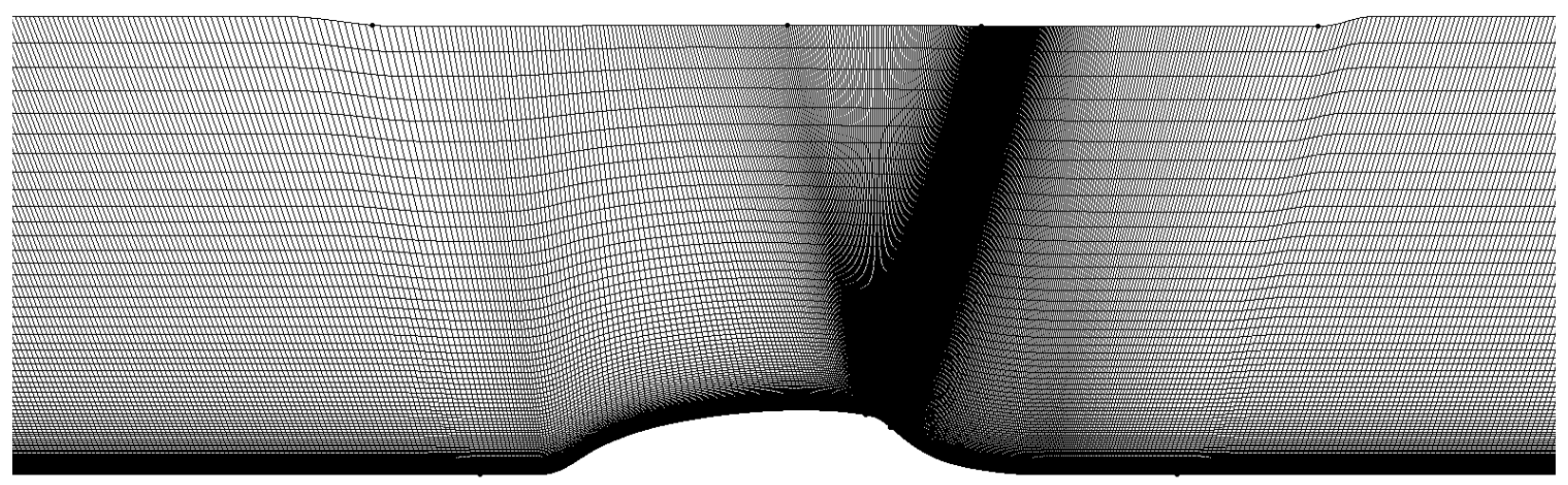

(b) struct4: modified upper-wall, contoured to match experimental pressure profile

Figure 5. Meshes used for ILES: $827 \times 100 \times 105$ points

Figure 8 (b) shows the variation of skin friction for the experiment, ILES and RANS. Once again, similar to the observation of Morgan et al., the higher $\theta / c$ results in a significantly higher $C_{f}$ in the fore-body of the hump. Whereas their peak $C_{f}$ was 0.01, ILES of the current flow conditions had a peak $C_{f}$ of 0.015 . Skin friction with RANS peaked at 0.018. Based on this plot, the point of reattachment for ILES was estimated to be at $x / c=1.25$, and $\approx 1.4$ for RANS. This is in contrast to the experimental result of $x / c \approx 1.1$ for $\theta / c$ $=0.77 \%$. There is, also, virtually no difference between both ILES meshes with regards to skin friction.

Mean streamwise and wall normal components of the flow velocity at different locations downstream of separation are compared in Fig. 9. Experimental data at $x / c=1.0,1.1,1.2$ and 1.3 are contrasted with ILES for both upper-wall definitions. Marginal differences between the upper-wall shapes are noticed only in the freestream. Figure 9 (a) shows the velocity profiles at $x / c=1.0$. ILES shows a stronger negative streamwise component compared to experiment. This trend continues at other locations downstream. Based on the experiment, for $\theta / c=0.77 \%$, the flow reattaches at $x / c \approx 1.1$, which is evident in Fig. 9 (b). For $\theta / c$ $=3 \%$, the flow continues to be separated until $x / c \approx 1.3$, where recovery starts, as in Fig. 9 (d). The point of reattachment based on these plots should lie between $x / c=1.2$ and 1.3 , which agrees with the value $x / c$ $=1.25$ from the $C_{f}$ plot in Fig. 8 (b).

In Fig. 10, mean Reynolds stress components $\langle u u\rangle,\langle v v\rangle$ and $\langle u v\rangle$ are compared at the same $x / c$ locations with experiment. $\langle u u\rangle$ and $\langle v v\rangle$ compare well at $x / c=1.0$ until $y / c=0.6$, above which the thicker inflow profile $(\theta / c=3 \%)$ tends to exhibit higher magnitudes than the thinner profile $(\theta / c=0.77 \%)$. A similar trend is observed for $\langle u u\rangle$ and $\langle u v\rangle$ at the downstream locations as well, whereas the wall normal component $\langle v v\rangle$ is significantly higher for the thicker profile throughout the boundary layer (except very close to the wall). The higher magnitude of Reynolds stresses conforms with the observation of a longer separation bubble. 


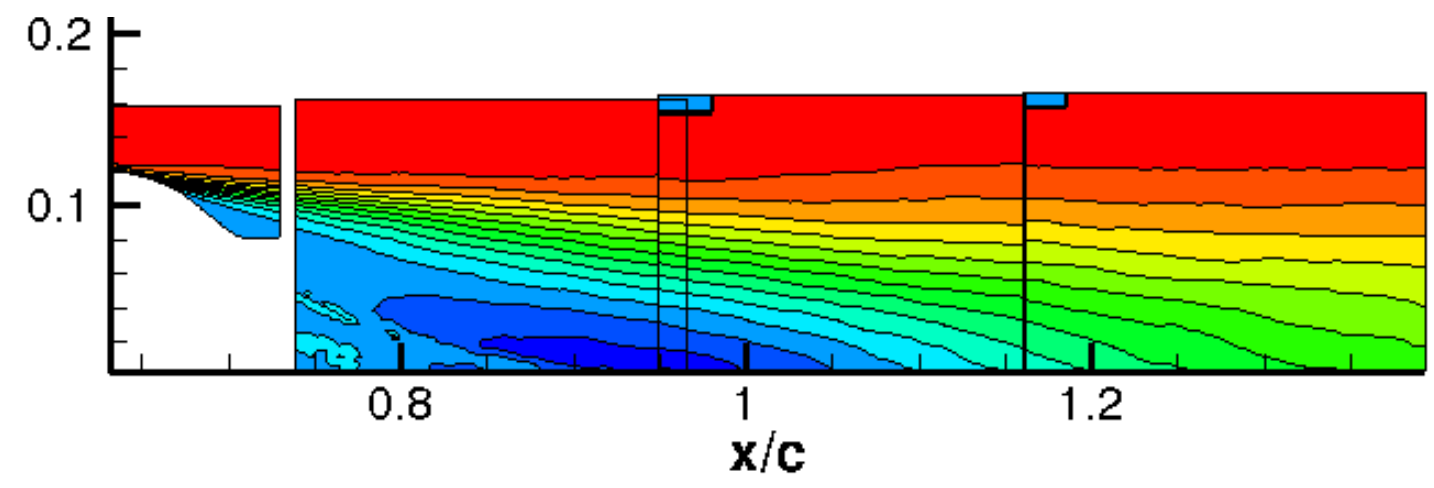

(a) PIV for $\operatorname{Re}_{c}=936,000$

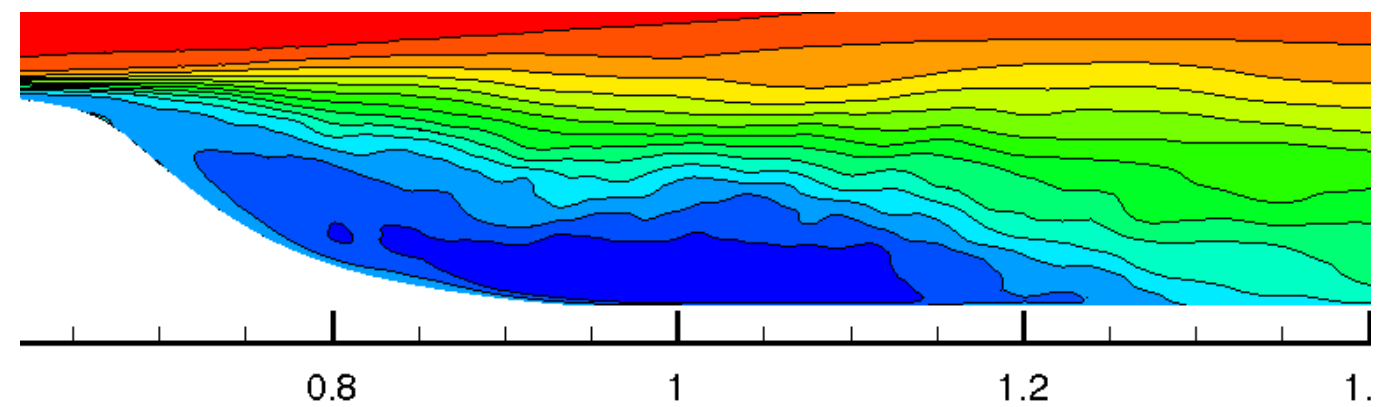

(b) ILES (struct1) for $\operatorname{Re}_{c}=47,500$

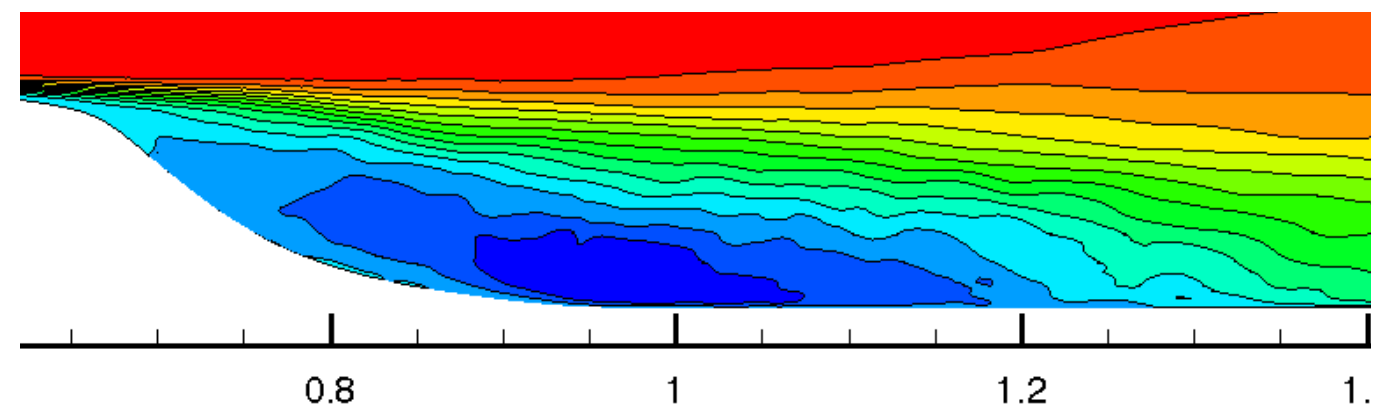

(c) ILES (struct4) $\operatorname{Re}_{c}=47,500$

Figure 6. Contours of $U / U_{\infty}$

Considering the differences in inflow profiles between the simulations and experiment, to make a better estimate of recovery, the axial location of reattachment for the experiment was shifted so as to coincide with ILES, and the mean profiles and Reynolds stresses downstream of that point were compared. These plots are shown in Figs. 11 and 12.

The overall trend of recovery shows very good agreement for both $\theta / c$ inflow profiles. The experiment with the thinner profile is slightly offset to the right, which is attributed to the differences in Reynolds numbers. But the shapes of both $U$ and $V$ match very well. Similarly, there is significantly improvement agreement of the Reynolds stresses, evident in Fig. 12. Here again, differences are attributed to the inflow conditions, but the trend of the stresses from ILES while progressing downstream conform with experiment very well.

\section{Conclusions}

As a part of the effort to improve turbulence models for separated flows, and considering the lack of high quality DNS for the wall-mounted hump configuration, direct simulations of flow with thicker inflow profiles have been conducted. By compromising on the $\theta / c$ ratio ( $3 \%$ for simulations vs $0.77 \%$ for experiment), 


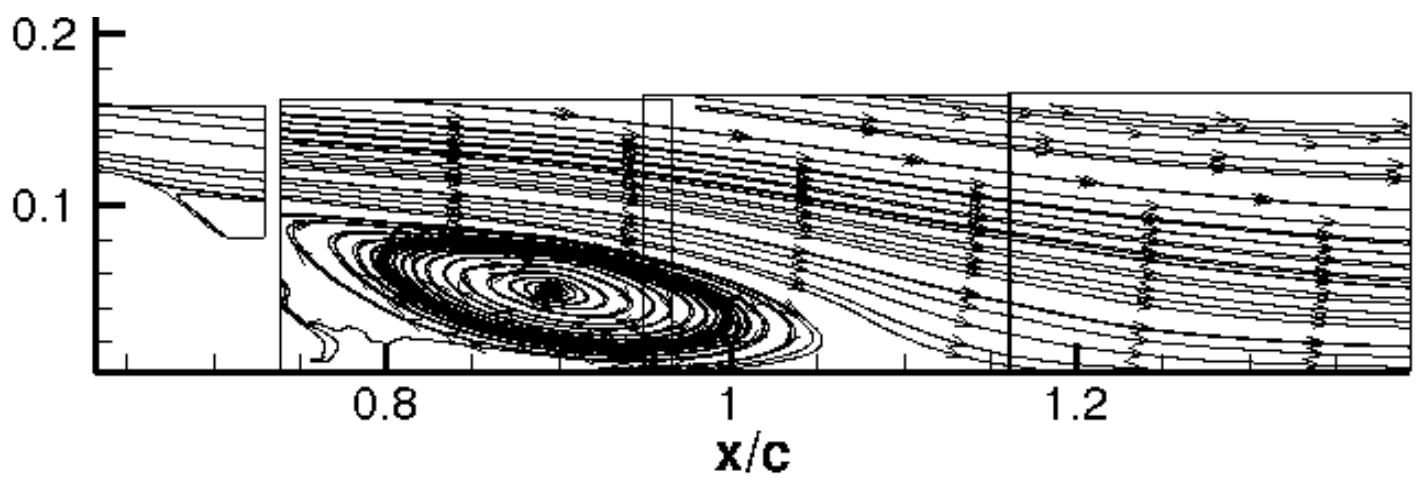

(a) PIV for $\operatorname{Re}_{c}=936,000$

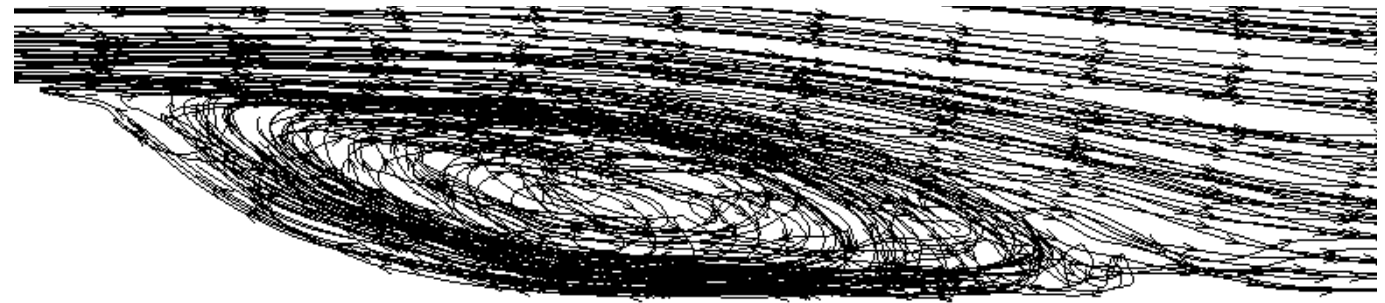

(b) ILES: struct1 $\left(\operatorname{Re}_{c}=47,500\right)$

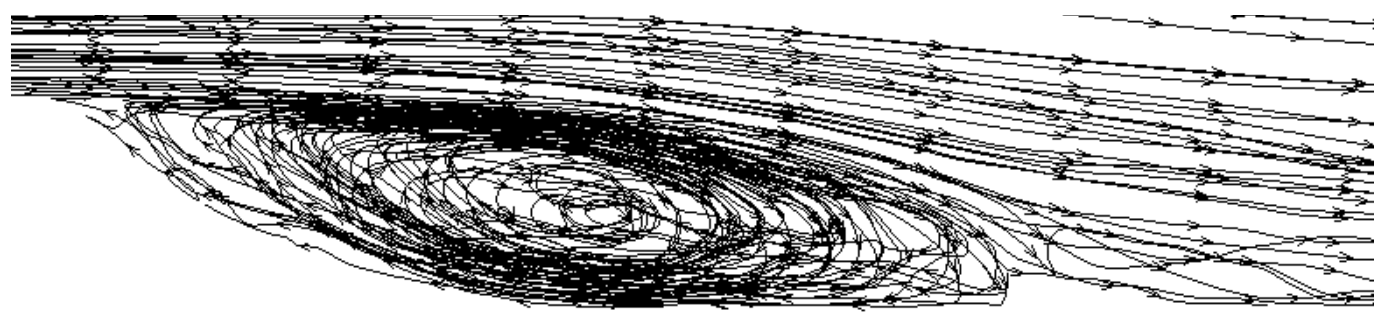

(c) ILES: struct4 $\left(\operatorname{Re}_{c}=47,500\right)$

Figure 7. Streamlines

DNS-like mesh resolutions, as well as spans of adequate widths, could be afforded.

Qualitative comparisons with experiment show an earlier point of separation for the higher $\theta / c$ simulations, and a later point of reattachment. The overall trends of pressure and skin friction variations over the surface agree, but the magnitudes are vastly different. The thicker inflow profiles result in significantly higher pressures in the fore-body of the hump, and lower pressures within the separation bubble.

Comparisons of the mean velocity profiles along different streamwise locations downstream of reattachment once again show differences in the bubble size, as well as recovery. Whereas the experimental reattachment point $(x / c)$ is 1.1 chord-lengths downstream of the leading edge of the hump for the thinner inflow, the simulation estimates it at $x / c=1.25$ for the thicker inflow. Reynolds stresses close to the wall tend to agree for both inflow conditions. But considering the differences in flow profiles, direct comparisons between experiment and simulations are inconsequential.

Instead, by shifting the streamwise location of reattachment to match with simulations, the trends of recovery, both in terms of mean velocity profiles and the Reynolds stresses, could be compared. Even though the magnitudes differ marginally because of the flow Reynolds numbers, the overall recovery trends in terms of mean velocities agree well for both inflow profiles. A similar agreement is also seen for the Reynolds stresses.

Two meshes with different upper-wall shapes were used, and comparisons of mean velocity profiles and Reynolds stresses show near identical values downstream of the point of separation. The skin friction variation along the surface is also the same. There are differences, though, in the pressure distribution over the surface of the hump, as well as within the separation bubble. But, this clearly has very little impact on 


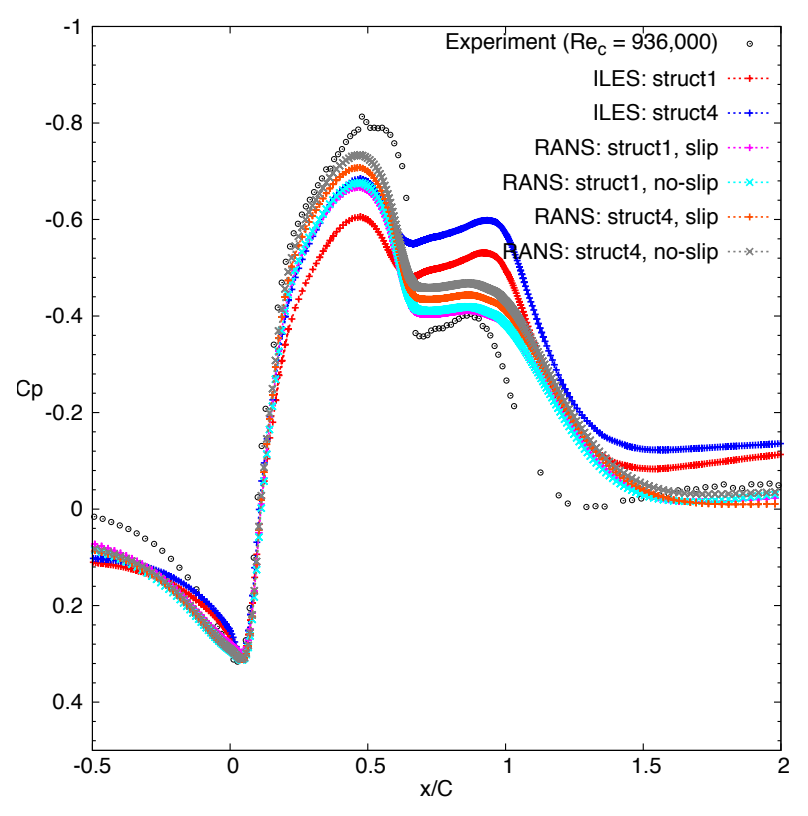

(a) $C_{p}$

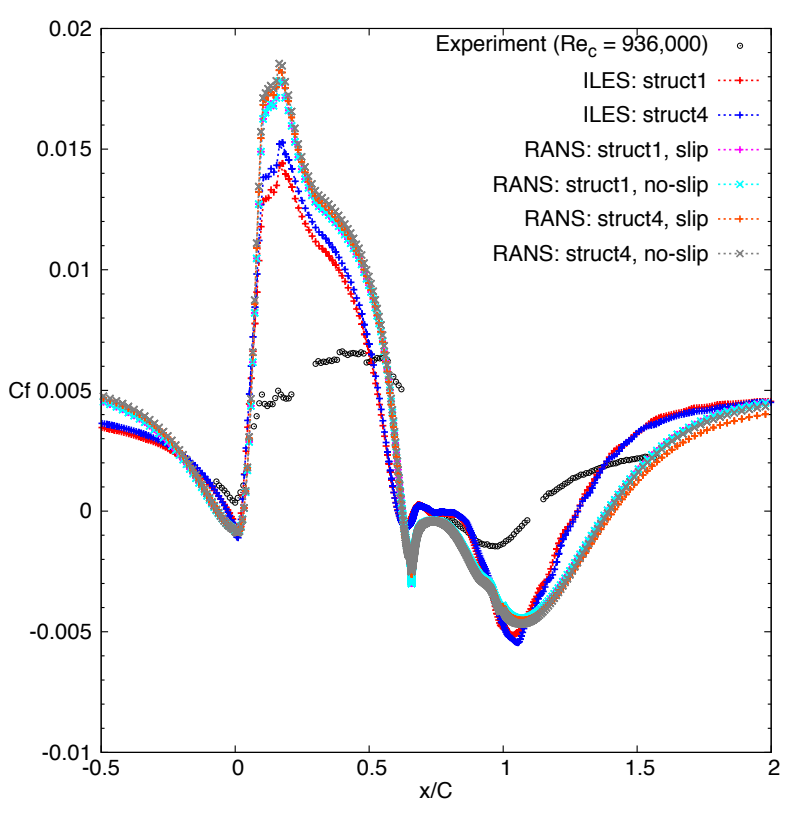

(b) $C_{f}$

Figure 8. Pressure and skin friction variation

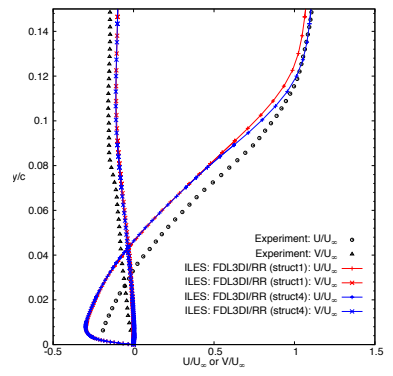

(a) $\mathrm{x} / \mathrm{c}=1.0$

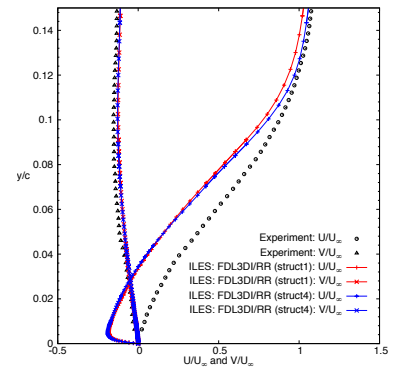

(b) $\mathrm{x} / \mathrm{c}=1.1$

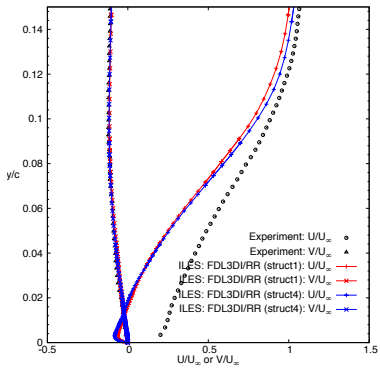

(c) $\mathrm{x} / \mathrm{c}=1.2$

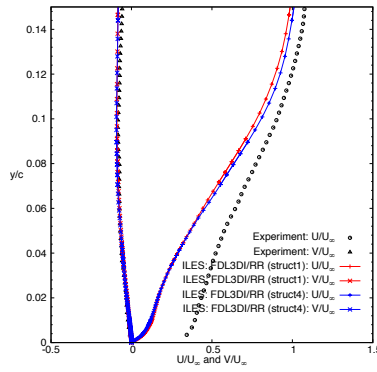

(d) $\mathrm{x} / \mathrm{c}=1.3$

Figure 9. Mean velocity profiles $\left(U / U_{\infty}\right.$ and $\left.V / U_{\infty}\right)$ at different streamwise locations

the characteristics of separation, be it the point of separation or reattachment, as well as recovery.

Based on the inferences from this initial test, additional simulations with inflows comparable to experiment are being conducted. Three-dimensionality effects of the side-walls, which the modified upper-wall shape was intended to account for, will also be investigated.

\section{Acknowledgements}

This research was supported by an appointment to the NASA Postdoctoral Program at Ames Research Center, administered by Oak Ridge Associated Universities through a contract with NASA. The authors are grateful for the support of NASA's Fundamental Aeronautics Program through the Aeronautical Sciences Project.

\section{References}

${ }^{1}$ Rumsey, C. L., "Proceedings of the 2004 Workshop on CFD Validation of Synthetic Jets and Turbulent Separation Control," Tech. Rep. NASA/CP-2007-214874, 2007.

${ }^{2}$ Rumsey, C. L., Gatski, T. B., III, W. L. S., Vatsa, V. N., and Viken, S. A., "Summary of the 2004 Computational Fluid Dynamics Validation Workshop on Synethetic Jets," AIAA Journal, Vol. 44, No. 2, 2006, pp. 194-207. 


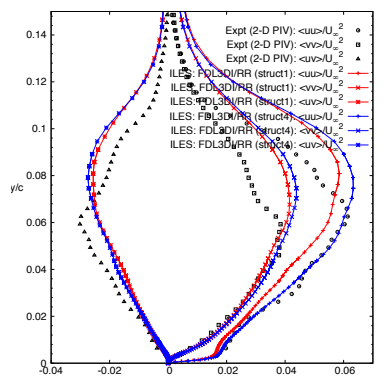

(a) $\mathrm{x} / \mathrm{c}=1.0$

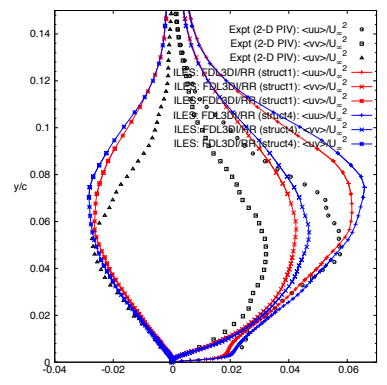

(b) $\mathrm{x} / \mathrm{c}=1.1$

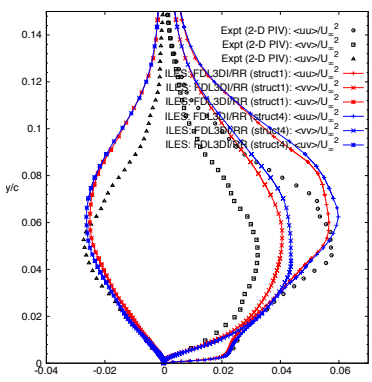

(c) $\mathrm{x} / \mathrm{c}=1.2$

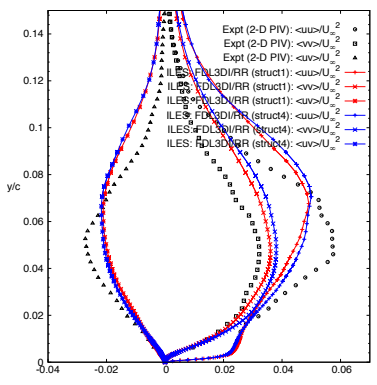

(d) $\mathrm{x} / \mathrm{c}=1.3$

Figure 10. Mean Re stress profiles $(<u u\rangle / U_{\infty}^{2},\langle v v\rangle / U_{\infty}^{2}$ and $\left.\langle u v\rangle / U_{\infty}^{2}\right)$ at different streamwise locations

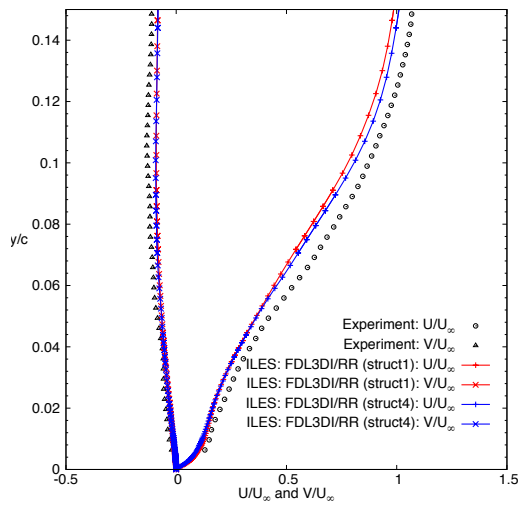

(a) $\mathrm{x} / \mathrm{c}=1.3$

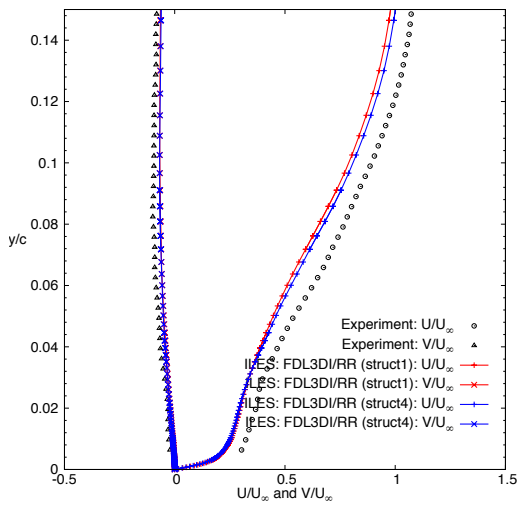

(b) $\mathrm{x} / \mathrm{c}=1.4$

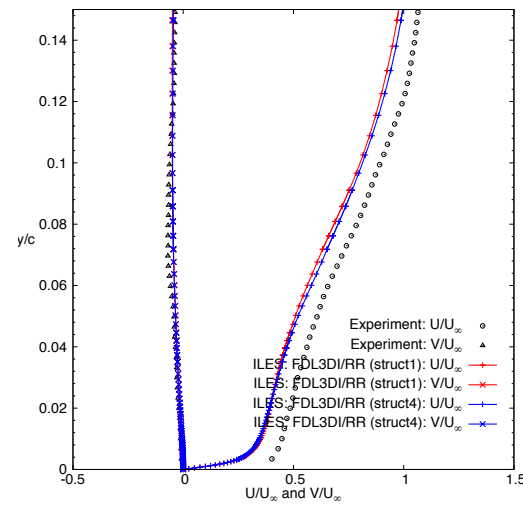

(c) $\mathrm{x} / \mathrm{c}=1.5$

Figure 11. Mean velocity profiles $\left(U / U_{\infty}\right.$ and $\left.V / U_{\infty}\right)$ at different streamwise locations; $x / c$ for experiment shifted to match ILES

${ }^{3}$ Rumsey, C. L., "Successes and Challenges for Flow Control Simulations (Invited)," 4th AIAA Flow Control Conference, No. 2008-4311, 2008.

${ }^{4}$ Seifert, A. and Pack, L. G., "Active Flow Separation Control on Wall-Mounted Hump at High Reynolds Numbers," AIAA Journal, Vol. 40, No. 7, 2002, pp. 1363-1372.

${ }^{5}$ Greenblatt, D., Paschal, K. B., Yao, C.-S., Harris, J., Schaeffler, N. W., and Washburn, A. E., "Experimental Investigation of Separation Control Part 1: Baseline and Steady Suction," AIAA Journal, Vol. 44, No. 12, 2006, pp. 2820-2830.

${ }^{6}$ Iaccarino, G., Marongiu, C., Catalano, P., and Amato, M., "RANS Modeling and Simulation of Synthetic Jets," $2 n d$ AIAA Flow Control Conference, No. 2004-2223, 2004.

${ }^{7}$ Capizzano, F., Catalano, P., Marongiu, C., and Vitagliano, P. L., "U-RANS Modelling of Turbulent Flows Controlled by Synthetic Jets," 35th AIAA Fluid Dynamics Conference and Exhibit, No. 2005-5015, 2005.

${ }^{8}$ Cui, J. and Agarwal, R. K., "CFD Validation of Turbulent Separation Control on a 2-D Hump (NASA Langley Workshop Validation: Case 3)," 35th AIAA Fluid Dynamics Conference and Exhibit, No. 2005-5013, 2005.

${ }^{9}$ Balakumar, P., "Computations of Flow over a Hump Using Higher Order Method with Turbulence Modeling," 43rd AIAA Aerospace Sciences Meeting and Exhibit, No. 2005-1270, 2005.

${ }^{10}$ Morgan, P. E., Rizzetta, D. P., and Visbal, M. R., "High-Order Numerical Simulation of Turbulent Flow over a WallMounted Hump," AIAA Journal, Vol. 44, No. 2, 2006, pp. 236-251.

${ }^{11}$ Bettinni, C. and Cravero, C., "Computational Analysis of Flow Separation Control for the Flow Over a Wall-Mounted Hump Using a Synthetic Jet," 45th AIAA Aerospace Sciences Meeting and Exhibit, No. 2007-0516, 2007.

${ }^{12} \mathrm{He}, \mathrm{C}$., Corke, T. C., and Patel, M. P., "Numerical and Experimental Analysis of Plasma Flow Control Over a Hump Model," 45th AIAA Aerospace Sciences Meeting and Exhibit, No. 2007-0935, 2007.

${ }^{13}$ Madugundi, D., Nagib, H., and Kiedaisch, J., "Evaluation of Turbulence Models Through Prediction of Separated Flows with and without Flow Control and Circulation Effects," 46th AIAA Aerospace Sciences Meeting and Exhibit, No. 2008-0567, 2008.

${ }^{14}$ Rumsey, C. L., "Reynolds-Averaged Navier-Stokes Analysis of Zero Efflux Flow Control over a Hump Model," Journal of Aircraft, Vol. 44, No. 2, 2007, pp. 444-452.

${ }^{15}$ Biswas, D., "Studies on Separation Control CFD Validation Test Case Based on a Higher Order LES Model," 3rd AIAA Flow Control Conference, No. 2006-3684, 2006.

${ }^{16}$ Krishnan, V., Squires, K. D., and Forsythe, J. R., "Prediction of Separated Flow Characteristics over a Hump," AIAA Journal, Vol. 44, No. 2, February 2006, pp. 252-262. 


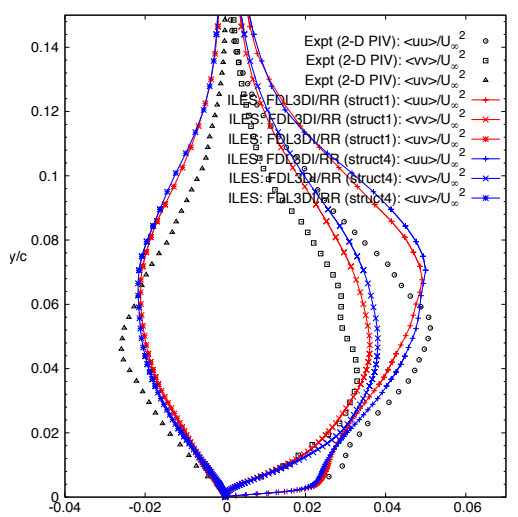

(a) $\mathrm{x} / \mathrm{c}=1.3$

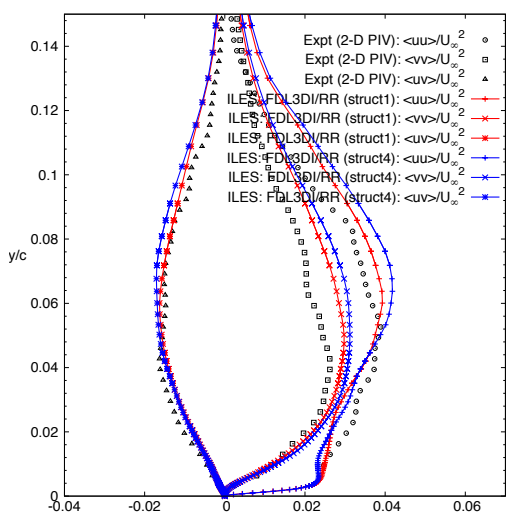

(b) $\mathrm{x} / \mathrm{c}=1.4$

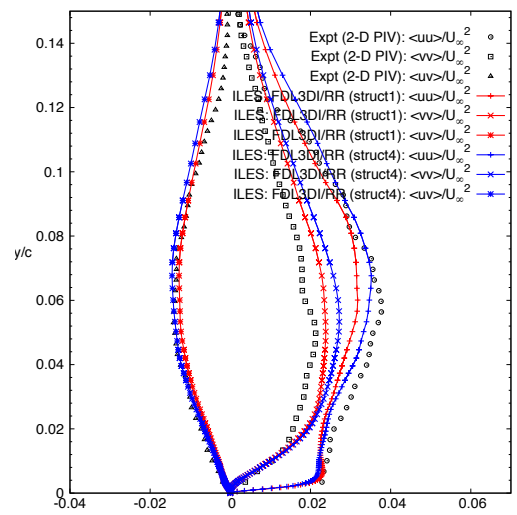

(c) $\mathrm{x} / \mathrm{c}=1.5$

Figure 12. Mean Re stress profiles $\left(\langle u u\rangle / U_{\infty}^{2},\langle v v\rangle / U_{\infty}^{2}\right.$ and $\left.\langle u v\rangle / U_{\infty}^{2}\right)$ at different streamwise locations

${ }^{17}$ Šarić, S., Jakirlić, S., and Tropea, C., "Computational Analysis of a Locally Forced Flow over a Wall-Mounted Hump at High-Re Number," Fourth International Symposium on Turbulence and Shear Flow Phenomena, edited by J. Humphrey, J. Eaton, R. Friedrich, N. Kasagi, M. Leschziner, and T. Gatski, June 2005, pp. 1189-1194.

${ }^{18}$ You, D., Wang, M., and Moin, P., "Large-Eddy Simulation of Flow over a Wall-Mounted Hump with Separation Control," AIAA Journal, Vol. 44, No. 11, November 2006, pp. 2571-2577.

${ }^{19}$ Franck, J. A. and Colonius, T., "Large-Eddy Simulations of Separation Control for Compressible Flow over a WallMounted Hump," 46th AIAA Aerospace Sciences Meeting and Exhibit, No. 2008-0555, 2008.

${ }^{20}$ Israel, D. M., Postl, D., and Fasel, H. F., "A Flow Simulation Methodology for Analysis of Coherent Structures and Flow Control," 2nd AIAA Flow Control Conference, No. 2004-2225, 2004.

${ }^{21}$ Hiller, S. J. and Seitz, P. A., "The Interaction Between a Fluidic Actuator and Main Flow Using SAS Turbulence Modeling," 3rd AIAA Flow Control Conference, No. 2006-3678, 2006.

${ }^{22}$ Morgan, P. E., Rizzetta, D. P., and Visbal, M. R., "Large-Eddy Simulation of Flow over a Wall-Mounted Hump," 43rd AIAA Aerospace Sciences Meeting and Exhibit, No. 2005-0484, 2005.

${ }^{23}$ Postl, D. and Fasel, H. F., "Direct Numerical Simulation of Turbulent Flow Separation from a Wall-Mounted Hump," AIAA Journal, Vol. 44, No. 2, February 2006, pp. 263-272.

${ }^{24}$ Johansson, T. G. and Davidson, L., "11th ERCOFTAC Workshop on Refined Turbulence Modelling," ERCOFTAC Bulletin, Vol. 69, 2005, pp. 28-34.

${ }^{25}$ Thiele, F. and Jakirlić, S., "12th ERCOFTAC Workshop on Refined Turbulence Modelling," ERCOFTAC Bulletin, Vol. 75, 2006, pp. 5-10.

${ }^{26}$ Noelting, S., Wessels, M., Keating, A., Satti, R., Li, Y., and Shock, R., "Lattice Boltzmann Simulations of the Flow over a Hump with Flow Control," 26th AIAA Applied Aerodynamics Conference, No. AIAA 2008-6721, 2008.

${ }^{27}$ Gan, S., Poondru, S., Ghia, U., and Ghia, K., "RANS and Large Eddy Simulation (LES) for Prediction of Separated Flow Characteristics for Flow Over a Wall-Mounted Hump," ASME/JSME 5th Joint Fluids Engineering Conference, No. FEDSM2007-37333, 2007, pp. 1405-1417.

${ }^{28}$ Šarić, S., Kniesner, B., Mehdizadeh, A., Jakirlić, S., Hanjalić, K., and Tropea, C., "Comparative Assessment of Hybrid LES/RANS Models in Turbulent Flows Separating from Smooth Surfaces," Advances in Hybrid RANS-LES Modelling, edited by S.-H. Peng and W. Haase, Vol. 97 of Notes on Numerical Fluid Mechanics and Multidisciplinary Design, Springer Berlin Heidelberg, 2008, pp. 142-151.

${ }^{29}$ Jakirlić, S., Manceau, R., Šarić, S., Fadai-Ghotbi, A., Kniesner, B., Carpy, S., Kadavelil, G., Friess, C., Tropea, C., and Borée, J., "LES, Zonal and Seamless Hybrid LES/RANS: Rationale and Application to Free and Wall-Bounded Flows involving Separation and Swirl," Numerical Simulation of Turbulent Flows and Noise Generation, edited by C. Brun, D. Juvé, M. Manhart, and C.-D. Munz, Vol. 104 of Notes on Numerical Fluid Mechanics and Multidisciplinary Design, Springer Berlin Heidelberg, 2009, pp. 253-282.

${ }^{30}$ Avdis, A., Lardeau, S., and Leschziner, M., "Large Eddy Simulation of Separated Flow over a Two-dimensional Hump with and without Control by Means of a Synthetic Slot-jet," Flow, Turbulence and Combustion, Vol. 83, No. 3, 2009, pp. 343370.

${ }^{31}$ Lyons, D. C., Peltier, L. J., Zajaczkowski, F. J., and Paterson, E. G., "Assessment of DES Models for Separated Flow From a Hump in a Turbulent Boundary Layer," Journal of Fluids Engineering, Vol. 131, No. 11, 2009.

${ }^{32}$ Ashton, N., Prosser, R., and Revell, A., "A hybrid numerical scheme for a new formulation of delayed detached-eddy simulation (DDES) based on elliptic relaxation," Journal of Physics: Conference Series, Vol. 318, No. 042043, 2011.

${ }^{33}$ Gritskevich, M. S., Garbaruk, A. V., Schütze, J., and Menter, F. R., "Development of DDES and IDDES formulations for the k- $\omega$ shear stress transport model," Flow, Turbulence and Combustion, Vol. 88, No. 3, 2012, pp. 431-449.

${ }^{34}$ Yoshio, M. and Abe, K., "Performance of Reynolds-Averaged Turbulence Models for Unsteady Separated Flows with Periodic Blowing and Suction," 18th International Symposium on Transport Phenomena, 2007, pp. 1359-1366. 
${ }^{35}$ Cappelli, D. and Mansour, N. N., "Performance of Reynolds Averaged Navier-Stokes Models in Predicting Separated Flows: Study of the Performance of Reynolds Averaged Navier-Stokes Models in Predicting Separated Flows: Study of the Hump Flow Model Problem," 31st AIAA Applied Aerodynamics Conference, No. AIAA 2013-3154, 2013.

${ }^{36}$ Gaitonde, D. V. and Visbal, M. R., "High-order schemes for Navier-Stokes equations: Algorithm and implementation into FDL3DI," AFRL-VA-WP TR-1998-3060, 1998.

${ }^{37}$ Morgan, P. E., Visbal, M. R., and Rizzetta, D. P., "A Parallel High-Order Flow Solver for Large-Eddy and Direct Numerical Simulation," 32nd AIAA Fluids Dynamics Conference and Exhibit, No. 2002-3123, 2002.

${ }^{38}$ Lele, S. K., "Compact Finite Difference Schemes with Spectral-like Resolution," Journal of Computational Physics, Vol. 103, 1992, pp. 16-42.

${ }^{39}$ Rizzetta, D. P., Visbal, M. R., and Blaisdell, G. A., "Application of a High-Order Compact Difference Scheme to LargeEddy and Direct Numerical Simulation," 30th AIAA Fluid Dynamics Conference, No. 1999-3714, 1999.

${ }^{40}$ Visbal, M. R., Morgan, P. E., and Rizzetta, D. P., "An Implicit LES Approach Based on High-Order Compact Differencing and Filtering Schemes (Invited)," 16th AIAA Computational Fluid Dynamics Conference, No. 2003-4098, 2003.

${ }^{41}$ Gaitonde, D. V. and Visbal, M. R., "Padé-Type Higher-Order Boundary Filters for the Navier-Stokes Equations," AIAA Journal, Vol. 38, No. 11, November 2000.

${ }^{42}$ Kawai, S. and Lele, S. K., "Localized artificial diffusivity scheme for discontinuity capturing on curvilinear meshes," Journal of Computational Physics, Vol. 227, 2008, pp. 9498-9526.

${ }^{43}$ Kawai, S., Shankar, S. K., and Lele, S. K., "Assessment of localized artificial diffusivity scheme for large-eddy simulation of compressible turbulent flows," Journal of Computational Physics, Vol. 229, 2010, pp. 1739-1762.

${ }^{44}$ Morgan, B., Duraisamy, K., Nguyen, N., Kawai, S., and Lele, S. K., "Flow physics and RANS modelling of oblique shock/turbulent boundary layer interaction," Journal of Fluid Mechanics, Vol. 729, 2013, pp. 231-284.

${ }^{45}$ Lund, T. S., Wu, X., and Squires, K. D., "Generation of Turbulent Inflow Data for Spatially-Developing Boundary Layer Simulations," Journal of Computational Physics, Vol. 140, 1998, pp. 233-258.

${ }^{46}$ Urbin, G. and Knight, D., "Large-Eddy Simulation of a Supersonic Boundary Layer Using an Unstructured Grid," AIAA Journal, Vol. 39, No. 7, July 2001, pp. 1288-1295.

${ }^{47}$ Morgan, B., Larsson, J., Kawai, S., and Lele, S. K., "Improving Low-Frequency Characteristics of Recycling/Rescaling Inflow Turbulence Generation," AIAA Journal, Vol. 49, No. 3, March 2011, pp. 582-597.

${ }^{48}$ Morgan, B. E., Large-Eddy Simulation of Shock/Turbulence Interactions in Hypersonic Vehicle Isolator Systems, Ph.D. thesis, Stanford University, 2012.

${ }^{49}$ Spalart, P. R., "Direct simulation of a turbulent boundary layer up to $\operatorname{Re}_{\theta}=1410$," Journal of Fluid Mechanics, Vol. 187, 1988, pp. 61-98

${ }^{50}$ Sekhar, S. and Mansour, N. N., "Implicit large-eddy simulations of zero-pressure gradient, turbulent boundary layer," AIAA Science and Technology Forum and Exposition 2015, 2015. 